Marquette University

e-Publications@Marquette

College of Nursing Faculty Research and

Publications

Nursing, College of

9-2019

\title{
Creation and Implementation of a Pediatric Advanced Practice Nurse Critical Care Fellowship Program
}

\author{
Bonnie Stojadinovic \\ Medical College of Wisconsin \\ Christine A. Schindler \\ Marquette University, christine.schindler@marquette.edu \\ Margaret Faut Callahan \\ Loyola University - Chicago \\ Maureen O'Brien \\ Regis University College of Nursing \\ Tom B. Rice \\ Medical College of Wisconsin
}

See next page for additional authors

Follow this and additional works at: https://epublications.marquette.edu/nursing_fac

Part of the Nursing Commons

\section{Recommended Citation}

Stojadinovic, Bonnie; Schindler, Christine A.; Callahan, Margaret Faut; O'Brien, Maureen; Rice, Tom B.; and Mikhailov, Theresa, "Creation and Implementation of a Pediatric Advanced Practice Nurse Critical Care Fellowship Program" (2019). College of Nursing Faculty Research and Publications. 624.

https://epublications.marquette.edu/nursing_fac/624 


\section{Authors}

Bonnie Stojadinovic, Christine A. Schindler, Margaret Faut Callahan, Maureen O'Brien, Tom B. Rice, and Theresa Mikhailov 
Marquette University

e-Publications@Marquette

\section{Nursing Faculty Research and Publications/College of Nursing}

This paper is NOT THE PUBLISHED VERSION; but the author's final, peer-reviewed manuscript. The published version may be accessed by following the link in the citation below.

Journal of Pediatric Health Care, Vol. 33, No. 5 (September-October 2019): 595-602. DOI. This article is (C) Elsevier and permission has been granted for this version to appear in e-Publications@Marquette. Elsevier does not grant permission for this article to be further copied/distributed or hosted elsewhere without the express permission from Elsevier.

\section{Creation and Implementation of a Pediatric Advanced Practice Nurse Critical Care Fellowship Program}

Bonnie Stojadinovic

Medical College of Wisconsin/Children's Hospital of Wisconsin, Milwaukee WI

Christine A. Schindler

Marquette University College of Nursing, Milwaukee, WI

Margaret Faut Callahan

Loyola University-Chicago, Chicago, IL.

Maureen O'Brien

Regis University College of Nursing, Denver, CO

Tom B. Rice

Medical College of Wisconsin/Children's Hospital of Wisconsin, Milwaukee WI

Theresa Mikhailov

Critical Care Pediatric Nurse Practitioner Program, Medical College of Wisconsin/Children's Hospital of Wisconsin, Milwaukee WI. 


\section{Keywords}

Pediatric advanced practice nurses, nurse practitioners, fellowship,education, training, program development

\section{Background}

Advanced practice registered nurses (APRNs) who begin their careers in the pediatric intensive care unit (PICU) may be challenged in this practice environment. Inadequate prior experience as a staff nurse, limited opportunities for clinical placements in the PICU during graduate education, and being in a fast-paced, highacuity practice environment without prior exposure to critically ill children are practice challenges in the PICU setting. The goal of postgraduate education training programs (fellowship programs) for the acute care pediatric nurse practitioner (ACPNP) is to prepare students to become beginner practitioners who can function effectively in the acute care setting within a few months of being hired, much like that of their physician counterparts who complete a fellowship.

The health care environment continues to be influenced by trends in national health care reform, shifts in the models for physician training, and the Accreditation Council for Graduate Medical Education resident duty hour restrictions. These emerging trends have given health care organizations the opportunity to evaluate their current care delivery and training models. It is expected that the demand for APRNs with specialty training will increase. The aim of this article is to describe our experience in the creation and implementation of a critical care pediatric nurse practitioner (CCPNP) fellowship training program at a large midwestern U.S. tertiary care center.

It is expected that the demand for APRNs with specialty training will increase.

When this fellowship was created, there were no known fellowships available for pediatric nurse practitioners (PNPs) interested in pediatric critical care. To meet the needs of these providers, a focused training program is required to provide specific preparation and competencies to practice to the full extent of the provider's license. A recent recommendation is for health care administrators to consider implementing fellowship training programs to assist nurse practitioners transitioning into specialty roles (Kells, Dunn, Melchiono, \& Burke, 2015). We used several online search engines to identify pediatric health care institutions with active advanced practice provider postgraduate fellowships. Our search in June 2017 identified fellowship programs in primary care, pediatric hematology/oncology, palliative care, neuro-critical care, and urgent care/emergency department. To our knowledge, this fellowship program was the first of its kind and seeks to provide postgraduate specialty training and education focused on the unique requirements of critically ill children and their families to help fill a knowledge gap when entering practice in this highly specialized practice environment.

\section{Purpose of The Fellowship Program}

The purpose of this program was to provide an effective and comprehensive fellowship in pediatric critical care that was mutually beneficial for both our organization and the CCPNP fellows. The objectives of our CCPNP fellowship for the fellows were to offer the opportunity to gain specialty experience, increase their marketability, enhance their leadership skills through doctoral-level coursework and educational opportunities, and enter the workforce with minimal additional onboarding required. The objectives we had regarding the organizations hiring these fellows after graduation were to decrease the expenses of orientation and reduce the burden of education specific to pediatric critical care.

Our program sought to train CCPNPs who were prepared to begin their careers as CCPNPs in the advancedbeginner to competent phases of development (Benner, 1984). This level of development is difficult to attain unless substantial, focused training can be given in a controlled, clinically focused environment such as a fellowship program. At our medical school and associated tertiary care center, the average orientation time 
varies from 5 to 10 months, depending on prior registered nurse (RN) experience and graduate clinical experiences. Therefore, we determined our CCPNP fellowship program objectives (Box 1).

BOX 1 Fellowship program objectives

- Identify and recruit individuals interested in obtaining focused training in pediatric critical care

- Offer an intense 1-year, postgraduate educational program in which the individual fellow is given the opportunity to spend focused, directed time with the diverse patient populations served by the pediatric intensive care unit (PICU) and cardiac intensive care unit

- Offer additional training in a limited number of elective rotations outside of the PICU (anesthesia, emergency department, hematology/oncology, cardiology, pulmonary, transport, sedation services, gastrointestinal, toxicology, and palliative care)

- Provide clinical and educational opportunities for the critical care pediatric nurse practitioner (CCPNP) fellow by partnering with the tertiary medical center and academic graduate nursing school

- Provide knowledgeable clinical preceptors (CCPNPs, certified pediatric nurse practitioners, and physicians) to mentor CCPNP fellows

- Assist CCPNP fellows with leadership interest to pursue doctoral education

- Assist CCPNP fellowship graduates in obtaining employment with pediatric critical care programs nationally that, because of size or financial constraints, cannot create their own fellowship programs or that may have limited time to offer for onboarding of a new graduate CCPNP

The purpose of our program was like that of physician fellowship programs: to train and prepare CCPNPs to function at organizations other than our own. Thus, our objectives may not align with those of organizations who wish to retain and hire their CCPNPs after graduation. For those programs who wish to pursue a fellowship with the expectation of hiring after completion, a CCPNP fellowship may allow them to give their fellows time to practice their clinical and research skills before actual hire, decrease onboarding time (1-3 months vs. the average of 5-10 months), and provide an increased return on investment by improving recruitment and retention through the expectation of an employment commitment (Kells et al., 2015). Additionally, there may be a cost savings for organizations if they hire CCPNPs at completion.

\section{Process and Resources for Program Development}

Several resources were used to get this CCPNP fellowship program functioning quickly. There was a paucity of literature about a CCPNP program in pediatrics when the program began (Kells et al., 2015, Scholtz et al., 2014; Varghese, Silvestri \& Lopez, 2012). In addition, we performed a strengths, weaknesses, obstacles, and threats (i.e., SWOT) analysis through phone conversations and e-mails with well-established successful adult critical care NP programs, surveyed programs that had graduate APRN fellows for several years, and had directors who had authored and presented seminars about their programs. On several occasions, we had conversations with the fellowship program directors at both Emory University and Vanderbilt University. Grabenkort, Meissen, Gregg, and Coopersmith (2017)) reiterate that structured critical care training programs for NPs and physician assistants allow those individuals to perform at their full scope of practice. Glassick's criteria for scholarship were used to design, implement, and assess this program (Glassick, Huber, \& Maeroff, 1997). This was a joint effort through the tertiary care medical center and medical school partner. The medical school partnered with its graduate nursing school partner to offer those individuals interested in a CCPNP fellowship position the opportunity to begin doctoral studies through the doctor of nursing practice or PhD program while completing fellowship training.

We met with key stakeholders at the medical school, hospital, and nursing school. We determined that support existed at all organizations during multiple planning sessions. Funding for the program was discussed with the 
medical school leadership, and one of the available CCPNP positions was converted into a funded fellowship position.

The current CCPNP members and the CCPNP leadership team determined the CCPNP team's time and resource availability for supporting the program. The CCPNP group was supportive of this endeavor, and some members offered to assist by functioning in the role of primary preceptor for the fellows. All members helped write goals and objectives for the various PICU rotations, and several members of the group collaborated with various specialty programs to create the unique goals and objectives necessary for a CCPNP fellow. The learning objectives for the CCPNP fellow for the specific clinical rotations are summarized in Box 2.

BOX 2 Fellowship learning objectives for specific clinical rotations

Competency-based educational goals for the rotation in the Cardiac Intensive Care Unit (CICU)

$\underline{\text { Objectives }}$

At the end of the rotation(s) in the $\mathrm{CICU}$, the critical care pediatric nurse practitioner (CCPNP) fellow will be able to the following:

1. Explain normal cardiac physiology

2. Explain the pre- and postoperative physiology of commonly seen congenital heart defects

3. Describe the physical examination, laboratory, and diagnostic study findings that aid in the assessment of the cardiovascular status of critically ill children

4. Select appropriate monitoring devices for care of the child with cardiac disease

5. Describe the common interactions of the respiratory and cardiac systems

6. Compare the impact of various ventilation modes (including spontaneous respiration) on cardiac function

7. Identify and select appropriate treatments for common dysrhythmias

8. Select and explain the rationale for use of common cardiac medications

9. Describe the common potential factors complicating the postoperative care of patients

10. Describe the medical management of heart failure, including types of mechanical support available (e.g., extracorporeal membrane oxygenation, Berlin Heart [Berlin, Germany], etc.)

$\underline{\text { Skills }}$

- Apply various methods of hemodynamic monitoring

- Interpret different waveforms for central venous pressure and intracardiac and arterial lines

- Determine the most appropriate choice of various types of mechanical support (e.g., extracorporeal membrane oxygenation, ventricular assist devices)

- Interpret laboratory studies related to the care of cardiac patients (e.g., arterial blood gases, B-type natriuretic peptide, mixed venous oxygenation, lactate)

- Interpret common electrocardiography results

- Interpret chest radiography results

- Select appropriate imaging modalities for patients with cardiac conditions (e.g., echocardiogram, cardiac magnetic resonance imaging)

Competency-based educational goals for rotation in the Surgical/Trauma PICU

$\underline{\text { Objectives }}$

At the end of the rotation(s) on the surgical/trauma PICU, the CCPNP fellow will be able to do the following:

1. Explain the most common diseases involving neurologic physiology encountered in the surgical PICU 
(traumatic brain injury, seizures, brain tumors, diabetes insipidus, syndrome of inappropriate diuretic hormone, stroke)

2. Explain the most common diseases involving respiratory physiology encountered in the surgical PICU (acute respiratory failure syndrome, respiratory viruses, pneumonia, asthma)

3. Explain the most common diseases involving gastrointestinal physiology encountered in the surgical PICU (gastrointestinal bleeding, liver failure, kidney failure/dialysis, pseudo-obstruction and bowel obstruction, acute abdomen, solid organ transplant)

4. Explain the most common diseases and issues involving the hematologic system encountered in the surgical PICU (deep vein thrombosis (stroke), pulmonary embolism, use of anticoagulation, use of blood products)

5. Explain systemic inflammatory response syndrome, sepsis, and septic shock

6. Explain rationale for the selection of appropriate antibiotics in the surgical PICU

7. Explain rationale for the selection of common laboratory and diagnostic studies and findings that aid in the assessment of common surgical issues of critically ill children

8. Explain the interaction of various physiologic systems in the case of trauma

9. Explain the role of early nutrition and rationale for enteral versus parenteral nutrition in the surgical critically ill child

10. Identify and understand the rationale for the most frequently used modes of mechanical ventilation in the surgical PICU

11. Identify and understand rationale for the placement and care of tracheostomy in the critically or chronically ill child

12. Select the appropriate monitoring devices for care of the child with disease requiring surgical intervention 13. Select and explain the rationale for the use of common cardiac, respiratory, neurologic, and hematologic medications

14. Describe the common potential factors complicating the postoperative care of patients.

$\underline{\text { Skills }}$

- Insertion of a tracheostomy tube in an established tracheostomy patient

- Hand mechanical ventilation

- Procedural sedation

- Procedures

o Peripheral intravenous line placement

o Arterial line placement

o Central line placement

o Lumbar puncture

o Intubation

o Ultrasonography

Competency-based educational goals for the rotation in the Medical/Oncology PICU

$\underline{\text { Objectives }}$

At the end of the rotation(s) on the medical/oncology PICU, the PNP will be able to do the following:

1. Explain normal pediatric pulmonary anatomy, structure, and physiology

2. Explain most common modes of mechanical ventilation used in the pediatric population (noninvasive, invasive, pressure control/pressure support)

3. Diagnose common respiratory problems and create an appropriate differential diagnosis and management plan

4. Explain basic cardiac anatomy, structure, and physiology 
5. Identify basic cardiac rhythms, including cardiac rhythm disorders

6. Explain the rationale for various types of fluids (e.g., normal saline, lactate ringers, $5 \%$ dextrose, $10 \%$ dextrose) and be able to choose appropriate fluids and calculate maintenance needs for pediatric patients requiring intravenous fluids

7. Explain the etiology and management of dehydration in the pediatric patient and calculate fluid deficits

8. Explain the nutrition monitoring needed in specific pediatric conditions

9. Explain the etiology and treatment of acute liver failure in children

10. Explain the etiology, pathophysiology, consequences, and treatment of the most common diseases of the hematologic system (anemia, sickle cell, oncologic, and bone marrow transplant) in critically ill children.

11. Explain the components of a work-up for an infectious disease in critically ill pediatric patients, including neonates

12. Select and explain the rationale for use of common antimicrobial/antifungal/antiviral medications

13. Explain systemic inflammatory response syndrome, sepsis, septic shock, diagnosis, diagnostics evaluation, and treatment

14. Explain etiology, pathophysiology, clinical presentation, and treatment of specific renal conditions

15. Explain and be able to articulate the critical steps in the approach to the poisoned patient (patient history, physical examination, initial management, diagnostic evaluation)

16. Explain commonly encountered genetic, metabolic, and endocrine disorders in the medical/oncologic intensive care unit

17. Explain the basic anatomy and physiology of the human nervous system

18. Explain the etiology, presentation, epidemiology, and pathophysiology associated with common neurologic conditions of children presenting to the medical intensive care unit (altered mental state, encephalopathy, seizures, spasticity, stroke)

19. Explain the pathophysiology, epidemiology, and etiology of the patient with encephalopathy

\section{$\underline{\text { Skills }}$}

Pulmonary/Respiratory Skills

- Suctioning of the airway (oral, nasal, or artificial airway)

- Oral/nasopharyngeal airway placement and monitoring

- Perform tracheostomy tube change and perform tracheostomy care

- Apply positive pressure using bag-valve mask

- Perform bag-mask ventilation

- Control the airway for intubation

- Perform the application of cricoid pressure

- Perform intubation/extubation

- Interpret continuous end-tidal carbon dioxide monitoring.

Fluids, Electrolytes, Nutrition, and Gastrointestinal Skills

- Calculation of maintenance fluids for hydration in the pediatric patient using the Holliday-Segar method.

- Calculate daily caloric intake of enteral nutrition per kg of weight

- Calculate daily fluid volume intake per kg of weight

- Calculate daily urine output per kg of weight

- Calculate fluid deficit in dehydration

- Place enteral access for nutrition (nasogastric, nasoduodenal, nasojejunal) delivery

- Use Corpak (Buffalo Grove, IL) device for nasojejunal tube placement 
Hematology/Oncology/Transplant Skills

- Calculate absolute neutrophil count

Infectious Disease Skills

- Perform lumbar puncture

- Obtain nasopharyngeal swabs for respiratory viral testing

Renal Skills

- Titration of continuous veno-venous hemofiltration and hemodialysis

Toxicology Skills

- Calculate anion gap

- Calculate the osmolar gap

- Access online medication resources

- Access online poison control/treatment resources

Genetic/Metabolic/Endocrine Skills

- Calculate anion gap

- Calculate the volume and rate of fluid resuscitation using the Children's Hospital of Wisconsin Diabetic Ketoacidosis protocol.

- Calculate fluid restriction or medication dosing in diabetes insipidus

- Interpret laboratory results for adrenocorticotropic hormone stimulation test

Neurology Skills

- Perform full neurologic examination

- Perform cranial nerve examination

- Perform lumbar puncture

- Interpret laboratory results of lumbar puncture

In 2015, the medical school leadership presented the proposal for the CCPNP fellowship program to the pediatric advanced practice nurse subcommittee group of the Society of Critical Care Medicine and the acute care special interest group of the National Association of Pediatric Nurse Practitioners. These meetings were key in disseminating information about the program. Individuals in attendance at both meetings acknowledged that a fellowship program would be important to the ongoing training and education of individuals interested in pediatric critical care. Program information was shared with several colleges and universities with ACPNP programs, social media sites, and students. Several potential candidates applied for the CCPNP fellowship, and an offer was extended to one candidate after Skype and in-person interviews.

We chose the initial candidate for this CCPNP fellowship based on several attributes we believe are important for a candidate to have, including resiliency, which was assessed with specific, targeted questions that we modified from the "50 Commonly Asked Interview Questions" in the "Professional Guide: Preparing for an Interview Guide" at the Center for Career Development at the Maxwell School of Citizenship and Public Affairs at Syracuse University (Syracuse University, n.d.; Box 3). We believe that resiliency is crucial, particularly in the 
inaugural year. Additional attributes a successful fellow should have include strong clinical decision-making skills, flexibility as programmatic changes occurred, and ability to advance from full mentoring to increasing autonomy after the first 6 months of fellowship.

BOX 3 Sample resiliency and behavioral-based interview questions

Remember to remain consistent and to avoid any discrimination; questions asked of one candidate should be asked of all candidates.

- Describe a situation in which you were able to use persuasion to successfully convince someone to see things your way.

- Describe a time when you were faced with a stressful situation that demonstrated your coping skills.

- Give me a specific example of a time when you used good judgment and logic in solving a problem.

- Give me an example of a time when you set a goal and were able to meet or achieve it.

- Tell me about a time when you had to use your presentation skills to influence someone's opinion.

- Give me a specific example of a time when you had to conform to a policy with which you did not agree.

- Tell me about a time when you had too many things to do and you were required to prioritize your tasks.

- Give me an example of a time when you had to make a split-second decision.

- What is your typical way of dealing with conflict? Give me an example.

- Tell me about a time you were able to successfully deal with another person even when that individual may not have personally liked you (or vice versa).

- Tell me about a difficult decision you've made in the last year.

- Give me an example of a time when something you tried to accomplish and failed.

- Give me an example of when you showed initiative and took the lead.

- Tell me about a recent situation in which you had to deal with a very upset customer or coworker.

- Tell me about a time when you missed an obvious solution to a problem.

- Describe a time when you anticipated potential problems and developed preventive measures.

- Tell me about a time when you were forced to make an unpopular decision.

\section{Organization and planning skills}

1. Describe a specific situation that illustrates how you set objectives to reach a goal.

2. Tell me about a time when you had to choose between two or more important opportunities. How did you go about deciding which was most important to you?

3. Tell me how you normally schedule your time in order to accomplish your day-to-day tasks.

4. Describe a time when you reorganized something to be more efficient. How did you do it?

5. Think of a time when you made important plans that were fouled up. How did you react? What did you do?

\section{Interaction and leadership}

1. Tell me about an event in your past that has greatly influenced the way you relate to people.

2. Give a specific example that best illustrates your ability to deal with an uncooperative person.

3. Some people have the ability to "roll with the punches." Describe a time when you demonstrated this skill.

4. Tell me when you had to work with someone who had a negative opinion of you. How did you overcome this?

5. Recall a time when you participated on a team. Tell me an important lesson you learned that is useful to you today.

\section{Assertiveness and motivation}

1. Describe several work standards that you have set for yourself in past jobs. Why are these important to 
you?

2. Tell me a time when you have experienced a lack of motivation. What caused this? What did you do about it?

3. Describe a situation that made you extremely angry. How did you react?

4. Tell me about a time that best illustrates your ability to "stick things out" in a tough situation.

\section{Cost, Benefits, And Goal Considerations}

When initiating such a program, the cost, benefits, and goals of the organization should always be considered. Our CCPNP fellowship, with the goal of training individuals to function elsewhere, gave us the benefit of creating a nationally recognized CCPNP training program. There was no decrease in other PNP clinical responsibilities or time commitment.

The benefits to our fellow were many. The clinical training hours were limited to 40 hours/week, offered Monday through Friday, to optimize exposure to educational opportunities and allow time to pursue doctoral education. The fellow received hands-on, focused clinical and didactic training in the care of critically ill children while having direct supervision and exposure to knowledgeable, fully functional CCPNPs. The fellow also received dedicated simulation training and additional educational experiences that he or she might not have been given as a new employee during orientation. For those programs that wish to hire a graduate of a CCPNP fellowship, it is expected that they will benefit by decreased onboarding time (1-3 months vs. the average of 510 months), decreased dedicated preceptor time, and a new employee with a higher entry-level clinical and research skill set.

Because cost is always a consideration, a budget and cost analysis should be performed first. This should include all forms of compensation for the fellow, as well as the expenses of initiating and maintaining the program. In our organization, the compensation package for the fellow included a 12-month appointment in which the individual received approximately $60 \%$ of a new graduate's salary and all regular employee benefits, including health care, insurance, vacation, sick time, and approved tuition reimbursement after the first 6 months of employment.

\section{Outcomes}

The selected initial CCPNP fellow showed progress in her clinical skills, expertise, and critical thinking skills. By 6 months, she was managing four or five patients and was able to take a full assignment of six to eight PICU patients with minimal support from her clinical preceptors before fellowship completion. Her final evaluations from both the CCPNPs and physicians showed ongoing growth from her initial evaluation at 2 months. Specific comments included improvements in her clinical skills, ability to manage difficult interactions, and ability to prioritize care and modify plans based on patient changes. Growth was tracked through formal quarterly written evaluations by preceptors, physician colleagues, and the program director. In addition, we conducted informal evaluations at least monthly with preceptors. Growth was measured by ongoing progress in meeting written goals and expectations, progressive ability to take more complex patients with higher acuity, and advancement to a full patient assignment. After leaving the fellowship, the individual was immediately hired into a busy pediatric emergency department. Although no formal follow-up with the hiring organization was obtained, the former fellow did report being able to easily transition into her first role after fellowship with minimal additional onboarding time. 
Specific comments included improvements in her clinical skills, ability to manage difficult interactions, and ability to prioritize care and modify plans based on patient changes.

The impact of this fellowship within the organization has been positive. The CCPNP team was excited about this program, vested in its success, and ready to move forward with the process of interviewing candidates for the next CCPNP fellowship position. Other programs at the medical school and tertiary medical center have shown interest in assessing the feasibility of this type of program for other specialty services and local adult programs. This program has been marketed through the medical school as an additional way we are committed to providing quality education for all medical providers receiving training at our organizations. Beginning dialogue about funding, candidate selection, and rudimentary requirements has occurred with a few other sites interested in initiating a similar program.

\section{Reflective Critique}

We believe that we were successful with many aspects of this program, including obtaining financial support for the CCPNP fellowship from the medical school, obtaining support and assistance in creating specific objectives from the current CCPNP team and involved specialty programs, and partnering with the graduate nursing school to offer the fellow initial coursework toward a doctoral degree. In addition, we were successful in marketing this program widely and creating interest in the fellowship.

Our second fellow started in April 2018, and we are in the process of finalizing candidate applications for the 2019 fellowship. We remain open to partnering with our adult nursing counterparts at the medical school to begin an adult CCNP fellowship program and to working with other critical care PNP programs to potentially begin similar programs at their organizations.

As with any new endeavor, there were some identified barriers and areas for improvement in subsequent years. Our initial timeframe for interviewing candidates was delayed because of budget approval. Another area we identified for improvement was the timing of the APRN certification, state licensure, and organizational credentialing. State RN licensure in our state (not compact license) was required before entering the fellowship program; thus, this issue caused delays. Advanced practice certification and APRN licensure timing is dependent on the expectations of the fellowship program and needs to be made clear to the candidate. For our program, certification and APRN licensure were expected to occur within the first 6 months of starting the program. However, at our institution, APRN credentialing is a longer, more complicated process than RN credentialing, which delayed the fellow's ability to provide direct patient care. These issues helped us identify future directions and the implications these issues can have for other programs interested in creating a CCPNP fellowship.

After looking at our first attempt at implementing a CCPNP fellowship program, we have a better understanding of next steps. We recommend beginning to recruit for the position 8 months before beginning a fellowship program and finalizing the recruitment process 6 months before the next candidate being hired. We recommend identifying key state licensure and organizational requirements before initiating a program like this. In addition, all appropriate background checks and credentialing packets should be completed, reviewed, and approved by all organizations the fellow will be affiliated with (both hospital and medical) before employment begins.

An additional barrier our initial fellow experienced was a delay in credentialing due to passing the acute care PNP certification before her start date. We anticipated that the candidate would obtain acute care PNP certification within the first 6 months of hire. However, she passed the examination 1 month before hire. This caused the credentialing requirements to change from what we originally planned for those of a fully certified ACPNP, delaying her credentialing process and ability to fully function in her new role for a few months. 
Finally, identification of the exact expectations of the CCPNP fellow is imperative. In our own organization, this was something we had assumed would be understood, because we currently have physician critical care fellows. After the first few months, we realized that many of the physicians were under the impression that the CCPNP fellow functioned like current providers regarding time and clinical assignment requirements. We realized that additional education about the role was needed. Role expectations were shared during faculty and physician critical care fellowship meetings, with questions addressed and additional education about the role provided.

To date, both formal and informal follow-up with the CCPNP fellowship leadership team, the fellow, the preceptors, and the CCPNP team have helped us realign our program goals. We have also learned how to best merge the demands of clinical competency with personal goals for doctoral education preparation by redesigning the clinical and classroom expectations, including the number of days needed for doctoral studies separate from clinical expectations. We believe that with each subsequent CCPNP fellow, we will continue to identify both positive outcomes and deficiencies of the program to allow for program enhancement. For organizations interested in pursuing a similar program, we recommend addressing the considerations listed in Box 4 early in the planning process.

We believe that with each subsequent CCPNP fellow, we will continue to identify both positive outcomes and deficiencies of the program to allow for program enhancement.

\section{BOX 4Important considerations}

- Financial support

o Who will provide it?

o What is the source of funding? Is it a sustainable revenue stream?

o What other compromises in relation to position modifications may need to be made (conversion of a regular pediatric nurse practitioner position, adding a position)?

\section{- Time and resource commitment}

o Who will be the point person?

o Does the division have buy-in from physician providers and other critical care pediatric nurse practitioners (CCPNPS)?

o Will other specialty programs support alternate clinical rotations?

- Flexibility to modify the planned curriculum based on individuals needs of the fellow and services involved.

- Credentialing

o Who credentials the CCPNP fellow?

o What is the anticipated time frame for the credentialing process?

o Special considerations by state (residency before starting, certification completed or in process, prescriptive privileges)

\section{Conclusion}

The demand for appropriately trained CCPNPs continues to rise. To date, limited formal postgraduate education and fellow training programs exist. We believe that the use of a structured formal training program such as a pediatric critical care PNP fellowship program offers new graduate acute care PNPs advanced knowledge and skills appropriate for beginning their careers in the PICU setting and allowing these individuals to function at the upper boundaries of their scope of practice much earlier during their careers. 


\section{References}

Benner, P. The Dreyfus model of skill acquisition applied to nursing. From novice to expert: Excellence and power in clinical nursing practice. Addison-Wesley, Menlo Park, CA; 1984: 13-34

Glassick, C.E., Huber, M.T., and Maeroff, G.I. Scholarship assessed: Evaluation of the Professoriate. Special report. Jossey Bass, San Francisco, CA; 1997

Grabenkort, R.W., Meissen, H.H., Gregg, S.R., and Coopersmith, C.M. Acute care nurse practitioners and physician assistants in critical care: Transforming education and practice. Society of Critical Care Medicine. 2017; 45: 1111-1114

Kells, M., Dunn, K., Melchiono, M., and Burke, P. Advanced practice nurse fellowships: Creating awareness, creating opportunities. Journal of Pediatric Health Care. 2015; 29: 297-301

Scholtz, A., King, K., and Kolb, S. The care model of the future: Supporting APRNs through an innovative transition to practice program. Journal of Pediatric Health Care. 2014; 28: 276-279

Syracuse University, Maxwell School of Citizenship and Public Affairs, Palmer Career Center. (n.d.). 50 Commonly asked interview questions. Syracuse, NY: Author. Retrieved from https://www.maxwell.syr.edu/uploadedFiles/Career/Commonly Asked Questions.pdf

Varghese, J., Silvestri, A., and Lopez, P. Reaching new heights: Development of the emergency department nurse practitioner fellowship program. Pediatric Emergency Care. 2012; 28: 6-7

\section{Biography}

Bonnie Stojadinovic, Director of Critical Care Pediatric Nurse Practitioner Fellowship Program, Medical College of Wisconsin/Children's Hospital of Wisconsin, Milwaukee WI.

Christine A. Schindler, Clinical Assistant Professor, Marquette University College of Nursing, Milwaukee, WI. Margaret Faut Callahan, Provost, Loyola University-Chicago, Chicago, IL.

Maureen O'Brien, Dean, Regis University College of Nursing, Denver, CO.

Tom B. Rice, Professor Emeritus, Medical College of Wisconsin/Children's Hospital of Wisconsin, Milwaukee WI. Theresa Mikhailov, Medical Director, Critical Care Pediatric Nurse Practitioner Program, Medical College of Wisconsin/Children's Hospital of Wisconsin, Milwaukee WI. 